\title{
1 Anti-biofilm multi drug-loaded 3D printed hearing aids
}

2

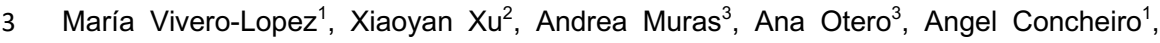

4 Simon Gaisford ${ }^{2,4}$, Abdul W. Basit ${ }^{2,4 ;}$, Carmen Alvarez-Lorenzo ${ }^{1 ; *}$, Alvaro Goyanes ${ }^{1,4 ; *}$

5

6

7

\section{7 *Corresponding authors}

18 Alvaro Goyanes, a.goyanes@FabRx.co.uk

19 Carmen Alvarez-Lorenzo, carmen.alvarez.lorenzo@usc.es

20

Abdul W. Basit, a.basit@ucl.ac.uk
21

22

23 


\section{Abstract}

Over $5 \%$ of the world's population has disabling hearing loss, which affects approximately one third of individuals over 65 years. Hearing aids are commonly used in this population group, but prolonged use of these devices may cause ear infections. We describe for the first time, the use of 3D printing to fabricate hearing aids loaded with two antibiotics, ciprofloxacin and fluocinolone acetonide. Digital light processing 3D printing was employed to manufacture hearing aids from two polymer resins, ENG hard and Flexible. The inclusion of the antibiotics did not affect the mechanical properties of the hearing aids. All multi-drug-loaded devices exhibited a hydrophilic surface, excellent blood compatibility and anti-biofilm activity against $P$. aeruginosa and $S$. aureus. Hearing aids loaded with ciprofloxacin $(6 \% \mathrm{w} / \mathrm{w})$ and fluocinolone acetonide $(0.5 \% \mathrm{w} / \mathrm{w})$ sustained drug release for more than two weeks and inhibited biofilm formation on the surface of the devices and bacteria growth in the surrounding medium. In summary, this work highlights the potential of vat photopolymerization $3 \mathrm{D}$ printing as a versatile manufacturing approach to fabricate high-fidelity patient-specific medical devices with anti-bacterial properties.

Keywords: hearing aids; drug-eluting medical device; 3D printing; sustained release; stereolithography; digital light processing; anti-biofilm medical devices; additive manufacturing 
Three-dimensional (3D) printing is an innovative and fast additive manufacturing technology based on the production of a physical object in a layer-by-layer manner from a computerized 3D model. The 3D models can be created by computer-aided design (CAD) software or obtained from imaging techniques (e.g. 3D scanner, computed tomography scan, magnetic resonance imaging) that capture images of the real object [1]. Due to its cost-efficiency, reproducibility and flexibility, 3D printing is forecast to be a disruptive technology in healthcare and drug delivery, changing manufacturing paradigms by allowing the fabrication of bespoke and individualized objects [2-4].

A wide variety of 3D printing technologies coexist today, and they can be classified depending on the nature of the material used (e.g. resins, plastics, ceramics), the deposition technology, and the mechanism used to form the layers or the characteristics of the final products [5]. Depending on the additive process involved, the American Society for Testing and Materials (ASTM) classifies these technologies into seven main categories: vat photopolymerization, material extrusion, directed energy deposition, powder bed fusion, binder jetting, material jetting and sheet lamination [6].

In the pharmaceutical industry, various 3D printing technologies included in the previously cited categories have been evaluated to prepare personalized medicines including fused deposition modelling (FDM) [7-9], semi-solid extrusion (SSE) [10, 11] and selective laser sintering (SLS) [12, 13]. Formulations with tailored drug release profiles [14, 15] and unique functions [16-19] have been previously introduced. However, the application of 3D printing has not stopped at the production of oral dosage forms. Multiple customized medical devices have been prepared by incorporating different drugs into the 3D printed structures for intrauterine [20], subcutaneous [21], vaginal [22], topical [1], intravesical [23], and oral [24, 25] drug delivery, and tissue scaffolds [26].

Vat photopolymerization 3D printing techniques, such as stereolithography (SLA), continuous liquid interface production (CLIP) and digital light projection (DLP) are processes where a vat of liquid photopolymerizable resin is selectively solidified using light irradiation (e.g. a laser, UV and visible light). Such 3D printing techniques provide high accuracy and superior resolution [27], and have been tested for biomedical and pharmaceutical applications [28], including the fabrication of polypills [29, 30], patientspecific implants [31], personalized devices [1], microneedles [32-34], dental devices [35], and hearing aids [36]. Hearing aids manufacturing is the paramount example of personalized medical devices that have benefitted from the development of 3D printing. In fact, more than $99 \%$ of the hearing aids that fit in user's ear canal are custom- 
manufactured by 3D printing [3, 37], mainly by vat photopolymerization technologies Manufacturers such as EnvisionTEC [36], Formlabs [38], and Sonova [39] have been employing 3D printing technology for production of shells for in-the-ear hearing aids to custom-made earpieces for behind-the-ear and receive-in-canal hearing aids as well ear products such as ear moulds and earbuds.

Around $5 \%$ of the population worldwide has disabling hearing loss, which affects approximately one third of people over 65 years [40]. Multiple causal agents are behind sensorineural and conductive hearing loss, but ear infection is a relevant cause of temporary hearing loss. Also, the prolonged use of hearing aids may alter the ear canal microbiota and increase the risk of fungal and bacterial otitis externa [41]. There is the need to prevent infections in hearing aids, however hearing aids with anti-biofilm properties have not yet been developed. In the case of ear infections, topical antibiotics are better than aural toilet (ear cleaning using dry mopping or irrigation) alone, and systemic or topical fluoroquinolones (e.g. ciprofloxacin, ofloxacin) are more effective than other types of antibiotics [42, 43]. It has also been reported that the use of ciprofloxacin in combination with fluocinolone acetonide is more effective than treatment with each drug individually for acute otitis media with tympanostomy tubes (AOMT) in children and is safe and well tolerated [44, 45].

Reports about manufacture of 3D printed devices with antibiofilm properties are limited [46]. To date, only a 3D printed device with nitrofurantoin to prevent the biofilm formation of S. aureus [47], an Ag-decorated 3D printed implant with antibiofilm activity against $E$. coli and S. aureus [48], and a 3D-printed PLA-collagen-minocycline-nanohydroxyapatite scaffold against S. aureus [49] were prepared. All of the above-mentioned examples were fabricated using FDM 3D printing technology, but the low resolution and mechanical properties of the produced objects may limit its applications in the production of complex devices such as the hearing aids.

To the best of our knowledge, there are no previous studies on 3D printed hearing devices that may act as drug release platforms in the outer-middle ear canal. As such, here we seek to explore the possibility of employing DLP 3D printing in fabrication of drug-loaded hearing aids for patients with ear infections. This novel drug-device combination product would avoid discontinuation of hearing aids use due to infections and may be even useful for normal hearing people suffering ear infections. A combination of ciprofloxacin and fluocinolone acetonide was chosen since it is commonly used in ear drops to address ear infections. The effect of the incorporation of two drugs on the printability and mechanical properties of the hearing aids and their capabilities to provide
Moved (insertion) [1]

Field Code Changed

Field Code Changed

\section{Deleted: ๆ}

Moved up [1]: In the case of ear infections, topical antibiotics are better than aural toilet (ear cleaning using dry mopping or irrigation) alone, and systemic or topical fluoroquinolones (e.g. ciprofloxacin, ofloxacin) are more effective than other types of antibiotics [40,41]. It has also been reported that the use of ciprofloxacin in combination with fluocinolone acetonide is more effective than treatment with each drug individually for acute otitis media with tympanostomy tubes (AOMT) in children and is safe and well tolerated [42, 43] 
controlled drug release were evaluated. Moreover, the efficacy of a_3D printed device to avoid the biofilm formation was evaluated against Pseudomonas aeruginosa (Gramnegative) and Staphylococcus aureus (Gram-positive), two avid biofilm formers involved in chronic ear infections $[50,51]$.

\section{Materials and methods}

\subsection{Materials}

Ciprofloxacin hydrochloride USP was purchased from Fagron (Spain) and fluocinolone acetonide (Ref. Mg93950) was from Guinama (Spain). Kudo 3DSR flexible resin (30$60 \%$ acrylate monomer; $35-69 \%$ acrylate oligomer; $1-5 \%$ photoinitiator) and Kudo 3DSR ENG hard resin (30-60\% acrylate monomer; 30-68\% acrylate oligomer; $1-10 \%$ photoinitiator; $1-10 \%$ pigment) were purchased from Kudo3D Inc. (USA). (3-(4,5Dimethylthiazol-2-yl)-2,5-diphenyltetrazolium bromide) (MTT) and orthophosphoric acid $\left(\mathrm{H}_{3} \mathrm{PO}_{4_{2}} 85 \%\right.$ ) were from Merck KGaA (Darmstadt, Germany); isopropyl alcohol and ethanol absolute $99.9 \%$ from VWR Chemicals (Fontenary-Sous-Bois, France); sodium chloride $\left(\mathrm{NaCl}_{2}\right.$, analytical grade) and tryptic soy broth (TSB) from Oxoid (UK); sodium hydroxide $\left(\mathrm{NaOH}_{2}\right.$ 98.5-100.5\%) from VWR Chemicals (Leuven, Belgium); bacto ${ }^{\mathrm{TM}}$ tryptone and bacto $^{\mathrm{TM}}$ yeast extract from Becton, Dickinson and Company (Le Pont de Claix, France); acetonitrile ( $\geq 99.9 \%$ purity, HPLC grade) from Scharlab S.L. (Barcelona, Spain). Water was purified using reverse osmosis (resistivity $>18 \mathrm{M} \Omega \cdot \mathrm{cm}$, MilliQ Millipore® Spain).

\subsection{Preparation of photopolymer solutions}

Flexible resin and ENG hard resin were used to prepare the photoreactive solutions. Flexible or ENG hard resins were mixed with $12 \%$ ciprofloxacin - $1 \%$ fluocinolone acetonide or $6 \%$ ciprofloxacin $-0.5 \%$ fluocinolone acetonide $(\mathrm{w} / \mathrm{w})$ to a total mass of $20 \mathrm{~g}$ (Table 1). The ciprofloxacin:fluocinolone acetonide mass ratio was fixed at 12:1, resembling the ratio in the marketed ear drops formulations [45]. The drugs were added to the resin solutions and the system was kept under magnetic stirrer for at least 2 hours until the drugs were apparently dissolved. The resulting photopolymer solutions were immediately loaded into the printer. Flexible resin-based devices and ENG hard resinbased devices were coded starting with the letter $\mathrm{F}$ or $\mathrm{H}$, respectively, followed by the content (in percentage) of each drug. Control formulations (without drug) were also printed and coded as FND or HND. 
Table 1. Composition of the 3D printed hearing aids. FND and HND are the codes for the blank hearing aids without drug.

\begin{tabular}{ccc}
\hline Hearing aid code & Resin & Drugs \\
\hline FND & Flexible & - \\
F6-0.5 & Flexible & $6 \%$ ciprofloxacin $-0.5 \%$ fluocinolone acetonide \\
F12-1 & Flexible & $12 \%$ ciprofloxacin $-1 \%$ fluocinolone acetonide \\
HND & ENG hard & - \\
H6-0.5 & ENG hard & $6 \%$ ciprofloxacin $-0.5 \%$ fluocinolone acetonide \\
H12-1 & ENG hard & $12 \%$ ciprofloxacin - 1\% fluocinolone acetonide \\
\hline
\end{tabular}

167

\subsection{D printing process}

A commercial Kudo3D Titan 2 HR 3D printer (Kudo3D Inc., USA) equipped with a digital light projector was utilized for printing the hearing aids. The printer allows fabrication of objects with high resolution and a layer thickness of 23 microns. The templates used to print the hearing aids were obtained from the moulds of the right ears of two volunteers and exported as a stereolithography file (.stl) into the 3D printer software (Kudo3D Print Job Software, Kudo3D Inc., USA). Additionally, circular discs (10 mm diameter x 1mm height) and rectangular-shape slabs $(10 \mathrm{~mm} \times 20 \mathrm{~mm} \times 1 \mathrm{~mm})$ were printed for microbiology studies and material testing. The printing time was 6 s per layer $(60$ s for the 1st layer) with a layer thickness of $25 \mu \mathrm{m}$. The post printing process consisted of a wash of $1 \mathrm{~min}$ in isopropyl alcohol and a cure process of $60 \mathrm{~min}$ at $60^{\circ} \mathrm{C}$ with UV-visible light (254-366 nm).

\subsection{Determination of hearing aids morphology}

Pictures of the devices were taken with a Nikon CoolpixS6150 camera with the macro option of the menu._The dimensions of the hearing aids were determined by measuring the device in the $X, Y$, and $Z$ axes. The images of the devices obtained from $X$-ray micro computed tomography were reconstructed, processed (as indicated in section 2.8), and saved in stl. format. The stl. files were then imported in the 3D printer software (Kudo3D Print Job Software, Kudo3D Inc., USA) to obtain the size of the device in the $\mathrm{X}, \mathrm{Y}$, and $\underline{Z}$ axes. The obtained values were then compared to the dimensions of the original $3 \mathrm{D}$ models.

\subsection{Scanning electron microscopy (SEM)}


3D printed slabs were sputter coated with iridium, and SEM images of the surface and cross-section were recorded in a Zeiss FESEM Ultra Plus microscope (Oberkochen, Germany).

\subsection{X-ray powder diffraction (XRPD)}

X-ray powder diffraction (XRPD) pattern of the drugs and the 3D printed slabs were recorded on a Philips type powder diffractometer fitted with a PW1710 control unit, a PW1820/00 goniometer and an Enraf Nonius FR590 generator. Spectra were made by measuring the scintillation response to $\mathrm{Cu} K \alpha$ radiation in the $2-50^{\circ} 2 \theta$ range, with a step size of $0.04^{\circ}$ and counting time of 6 s per step.

\subsection{Thermal analysis}

Differential scanning calorimetry (DSC) measurements of the drugs and the 3D printed slabs were performed using a differential scanning calorimeter Q200 DSC (TA Instruments, USA) previously calibrated with indium. The samples were accurately weighed in a $40 \mu \mathrm{L}$ aluminum pan, and then heated from 25 to $50^{\circ} \mathrm{C}$, cooled to $0^{\circ} \mathrm{C}$ and then heated to $300^{\circ} \mathrm{C}$ at a scanning rate of $10^{\circ} \mathrm{C} / \mathrm{min}$ in nitrogen atmosphere $(50 \mathrm{~mL} / \mathrm{min})$.

\section{$\underline{2.8 X \text {-ray micro computed tomography (Micro-CT) }}$}

A high-resolution X-ray micro computed tomography (Micro-CT) scanner (SkyScan1172,

Bruker-microCT, Kontich, Belgium) was used to visualise the internal structure of the hearing aids as in a previous study [13]. Each image was acquired by rotating the object through $180^{\circ}$ with a frame averaging of 4 and a $0.5^{\circ}$ rotation step using medium camera resolution (2000 x 1048 pixels). NRecon software (Version 1.7.0.4, Bruker-microCT, Kontich, Belgium) was used to reconstruct the images and the reconstructed images were processed using the software CTVox (version 2.3.2.0) to create the images. The software CT Analyzer (CTan version 1.16.4.1) was used to analyse the collected the data and calculate porosity and dimensions of the devices.

\section{$\underline{2.9 \text { Water contact angle }}$}


Water contact angles on $3 \mathrm{D}$ printed slabs were recorded for $10 \mathrm{~s}$ after the deposition of a water drop $(5 \mu \mathrm{L})$ taking one image per second. The measurements were carried out in triplicate using a Phoenix-300 plus video-based optical goniometer (SEO, Korea).

\subsection{Mechanical evaluation of the slabs}

$3 D$ printed slabs $(10 \mathrm{~mm} \times 20 \mathrm{~mm} \times 1 \mathrm{~mm})$ were tested in triplicate at room temperature in a TA.XT Plus Texture Analyzer (Stable Micro Systems, Ltd., UK) fitted with a $30 \mathrm{Kgf} \mathrm{load}$ cell. In each test, the slab was fixed to the upper and lower clamps with a gap of $6 \mathrm{~mm}$. The tensile test was carried out at a crosshead speed of $0.1 \mathrm{~mm} / \mathrm{s}$ until rupture. The stress-strain data were converted to engineering strength (force per cross-sectional area) versus the engineering strain (the change in active length divided by the original length), and the Young's modulus was calculated from the slope of the straight-line portion, as follows:

$$
E=\frac{F / A_{0}}{\Delta L / L_{0}} \quad \text { Eq. (1) }
$$

Where $E$ is the Young's modulus, $F$ is the force applied on the sample under tension, $A_{0}$ is the cross-sectional area, $\Delta \mathrm{L}$ is the change in length of the sample and $\mathrm{L}_{0}$ is the original length of the sample.

\subsection{Hemolytic activity}

The hemolysis assay was carried out in accordance with the direct test described in ISO10993-4 [52]. Fresh blood from anonymized healthy donors (Galician Transfusion Center, Spain) was diluted 1:30 (v/v) in $\mathrm{NaCl} 0.9 \%$ aq. medium. Aliquots $(1 \mathrm{~mL})$ were poured in Eppendorf tubes to which pieces of the slabs were added $\left(40-80 \mathrm{~mm}^{2}\right.$ side; 50-90 mg). Diluted blood without samples was used as negative control. The positive control was prepared adding $100 \mu \mathrm{L}$ of Triton X-100 $4 \%$ to tubes containing diluted blood. All tubes were incubated for $60 \mathrm{~min}$ in a Mini-Shaker (100 osc/min) at $37^{\circ} \mathrm{C}$ and then they were centrifuged (Sigma 2-16P; Sigma Laboratory Centrifuges, Germany) at $10,000 \mathrm{~g}$ for $10 \mathrm{~min}$. The supernatants $(150 \mu \mathrm{L})$ were transferred to 96 -well plate and the absorbance was recorded at $540 \mathrm{~nm}$ (FLUOstar Optima; BMG Labtech, Germany). Hemolysis percentage was calculated as follows:

Hemolysis $(\%)=\frac{A b s_{\text {sample }}-A b s_{\text {negative control }}}{A b s_{\text {positive control }}-A b s_{\text {negative control }}}$

Eq. (2) 


\subsection{Anti-biofilm properties}

Bacterial strains and growth conditions. The Gram-negative Pseudomonas aeruginosa PAO1 (Lausanne sub-line, donated by M. Cámara, Univ. of Nottingham) and the Grampositive bacteria Staphylococcus aureus ATCC25923 (ATCC, Manassas, VA, USA) were routinely cultured at $37{ }^{\circ} \mathrm{C}$ in Luria-Bertani Broth (LB) and $1 \%$ Tryptic Soy Broth (TSB-1), respectively.

Culture media preparation. LB (10 g/L of tryptone, $5 \mathrm{~g} / \mathrm{L}$ yeast extract, $10 \mathrm{~g} / \mathrm{L} \mathrm{NaCl}$ ) and TSB-1 (30 g/L TSB Scharlau, $5 \mathrm{~g} / \mathrm{L} \mathrm{NaCl}$ ) were prepared in distilled water. Culture media were magnetically stirred at $200 \mathrm{rpm}$ until complete dissolution and autoclaved $\left(121^{\circ} \mathrm{C}\right.$, $15 \mathrm{~min})$.

Amsterdam Active Attachment (AAA) model preparation. Bacterial biofilms were grown on dried materials submerged in culture medium using a modified AAA-model [53, 54] assembled with the tested materials and microscope glass coverslips $(18 \times 18 \mathrm{~mm}$, submerged surface $3.80 \mathrm{~cm}^{2}$ ) as controls. The coverslips were carefully placed in the silicone supports (borosilicate glass Menzel-Glaser, Thermo Scientific) of special metallic covers for 12-wells cell culture plates (Figure S1, Supplementary Material) with the help of a scalpel and tongs before being autoclaved $\left(121^{\circ} \mathrm{C}, 1 \mathrm{~atm}, 15 \mathrm{~min}\right)$. The sterilized covers were dried in an oven at $60{ }^{\circ} \mathrm{C}$ for $12-24 \mathrm{~h}$. The tested slabs were disinfected by immersion in ethanol $70^{\circ}$ for 5 seconds and then placed in the autoclaved AAA-model covers (Figure S1, Supplementary Material). The covers with the mounted materials were left to dry for one hour at room temperature in a biological safety cabinet before being submerged in 12-wells cell-culture plates containing $4 \mathrm{~mL}$ of culture media inoculated with the corresponding bacterial species.

Pre-inoculum and inoculum preparation. Pre-inocula were prepared by inoculating sterile Erlenmeyer flasks containing $10-\mathrm{mL}$ of culture medium with a colony of the corresponding bacterial pathogen. Flasks were incubated at $37{ }^{\circ} \mathrm{C}$ for $12 \mathrm{~h}(P$. aeruginosa) or $24 \mathrm{~h}$ (S. aureus) at $100 \mathrm{rpm}$. After incubation, optical density was measured at $600 \mathrm{~nm}$ (UV/Vis spectrophotometer Thermo Scientific Helios Omega) and adjusted to 0.05 (S. aureus) or 0.01 ( $P$. aeruginosa) by dilution with the corresponding culture medium in sterile $50 \mathrm{~mL}$ Falcon ${ }^{\circledR}$ tubes. The required dilutions were $1 / 326$ for $P$. aeruginosa and $1 / 125$ for $S$. aureus. The inocula were gently homogenized and distributed in the 12-wells cell culture plates $(4 \mathrm{ml}$ per well). All procedures were performed in a biological safety cabinet.

Plates preparation and incubation. All materials were tested in triplicate and incubated at $37^{\circ} \mathrm{C}$ for 12 and 48 hours for $P$. aeruginosa and $S$. aureus respectively. The $S$. aureus 
medium was changed every 12 hours by moving the models to new cell-culture plates previously filled with $4 \mathrm{~mL} /$ well of fresh TSB-1. In all cases, after each media exchange period, the absorbance of the culture medium was measured at $600 \mathrm{~nm}$ (UV/Vis spectrophotometer Thermo Scientific Helios Omega) after homogenization in order to assess planktonic bacterial growth.

Biofilm susceptibility. After the incubation period at $37{ }^{\circ} \mathrm{C}$, the viability of the bacterial biofilms was analyzed using MTT assay modified as previously reported $[55,56]$. For this purpose, slabs and coverslips were individually placed in sterile tubes containing $3.6 \mathrm{~mL}$ of PBS $\left(6.4 \mathrm{~g}\right.$ of $\mathrm{NaCl}, 0.16 \mathrm{~g}$ of $\mathrm{KCl}, 1.152 \mathrm{~g}$ of $\mathrm{NaHPO}_{4}$ and $0.192 \mathrm{~g}$ of $\mathrm{KH}_{2} \mathrm{PO}_{4}$ for 500 $\mathrm{mL}$ ) and then sonicated for $15 \mathrm{~min}$ in order to detach and homogenize the biofilms. After that, MTT solution $(5 \mathrm{mg} / \mathrm{mL}, 400 \mu \mathrm{L})$ was added to each tube, and the tubes were incubated at $37^{\circ} \mathrm{C}$ for $30 \mathrm{~min}$. The half of the MTT-containing PBS medium was removed and replaced with acid isopropanol ( $5 \%$ ( $\mathrm{vol} / \mathrm{vol}) 1 \mathrm{M} \mathrm{HCl}$ in isopropanol). After 5-min vortexing, aliquots $(1 \mathrm{~mL})$ of the medium were taken and their absorbance measured at 570 nm (UV/VIS spectrophotometer Thermo Scientific Helios Omega). PBS medium without samples was treated in the same way and used as a blank.

\subsection{Drug release studies}

Drug release studies were carried out from the 3D printed F6-0.5 and H6-0.5 (Table 1) discs (10 mm diameter $x 1 \mathrm{~mm}$ height and $0.1 \mathrm{~g}$ average weight) in $4 \mathrm{~mL}$ of $50 \%(\mathrm{v} / \mathrm{v})$ ethanol and $50 \%(\mathrm{v} / \mathrm{v})$ water for 67 days at $37^{\circ} \mathrm{C}$ and $400 \mathrm{rpm}(\mathrm{n}=4)$. The tubes were protected from light to avoid drug degradation. Blank (without drug) 3D printed discs were used as control. At predetermined time intervals, $500 \mu \mathrm{L}$ aliquots were withdrawn from each tube and replaced with the same volume of the release medium. The samples were filtered through a $0.22 \mu \mathrm{m}$ Nylon filter and the concentration of each drug was then determined using a JASCO (Tokyo, Japan) HPLC (AS-4140 Autosampler, PU-4180 Pump, LC-NetII/ADC Interface Box, CO-4060 Column Oven, MD-4010 Photodiode Array Detector), fitted with a C18 column (Waters Symmetry C18, $5 \mu \mathrm{m}, 4.6 \times 250 \mathrm{~mm}$ ) and operated with ChromNAV software (ver. 2, JASCO, Tokyo, Japan). The mobile phase consisted of $45 \%(\mathrm{v} / \mathrm{v}) 0.1 \%$ orthophosphoric acid $(\mathrm{pH} 2.2)$ and $55 \%(\mathrm{v} / \mathrm{v})$ acetonitrile at $30^{\circ} \mathrm{C}$, and the flow rate was kept at $0.9 \mathrm{~mL} / \mathrm{min}$. The injection volume was $20 \mu \mathrm{L}$ and the eluent was detected at a wavelength of $246 \mathrm{~nm}$ with a total run time of $10 \mathrm{~min}$ (retention time of ciprofloxacin and fluocinolone acetonide was $1.82 \mathrm{~min}$ and $7.21 \mathrm{~min}$, respectively). Ciprofloxacin and fluocinolone acetonide contents in the samples were calculated from a validated calibration curve. The standard solutions were $2.5-200 \mu \mathrm{g} / \mathrm{mL}$ 
of ciprofloxacin and $0.5-60 \mu \mathrm{g} / \mathrm{mL}$ of fluocinolone acetonide in $50 \%(\mathrm{v} / \mathrm{v})$ ethanol and 50 $\%(\mathrm{v} / \mathrm{v})$ water. At the end of the release test, all discs were dried in an oven $\left(37^{\circ} \mathrm{C}, 72\right.$ h) and weighed to determine the mass loss over the release time, and SEM images were obtained.

Drug release data was fit to the square root kinetics (Higuchi equation):

$Q=k_{H} \cdot t^{\frac{1}{2}} \quad$ Eq. (3)

Where $Q$ is the amount of drug released over time $t$ and $k_{H}$ is the Higuchi rate constant.

\subsection{Statistical analysis}

The effects of formulation composition on hemolysis and biofilm activity were analysed using ANOVA and multiple range test (Statgraphics Centurion 18, StatPoint Technologies Inc., Warrenton VA).

\section{Results and discussion}

\subsection{DLP 3D printing}

As everyone's ear anatomy is unique and different, hearing aids are one of the customized medical devices that are extensively manufactured via vat photopolymerization-based technology as a result of its high resolution and great efficiency. Compared with other manufacturing methods such as moulding, 3D printing was found to be cost effective when the production volume is low and avoids the need of making moulds, which requires extra costs on machinery, material and labour [57]. We successfully demonstrated the possibility of manufacture hearing aids loaded with two drugs, ciprofloxacin and fluocinolone acetonide, via DLP 3D printing. Previously, SLA has been utilized to produce polyprintlets (3D printed polypills) where multiple drugs were incorporated within a single pill but physically separated in different compartments [29, 30]. So far, there have not been examples of using photopolymerization-based 3D printing techniques to produce drug delivery systems that combine more than one drug incorporated at the same time in the same resin. Herein we demonstrated the possibility of preparing multi drug-loaded medical devices for personalised therapy.

Prior to the printing process, pure ciprofloxacin and fluocinolone acetonide were readily dispersed (apparently dissolved) in the Flexible or ENG hard resins under magnetic stirring. All the devices were successfully fabricated similar to the 3D scan model with 
smooth surface finish and good consistency in dimension regardless of drug loadings (Figure 1). The fabricated hearing devices appeared the same colors as the used resins, yellow for ENG hard (Figure 1B) and black for Flexible (Figure 1C). With the drug loading of ciprofloxacin-fluocinolone acetonide increasing, from $6 \%-0.5 \%$ to $12 \%-1 \%$, the density of colour slightly decreased.

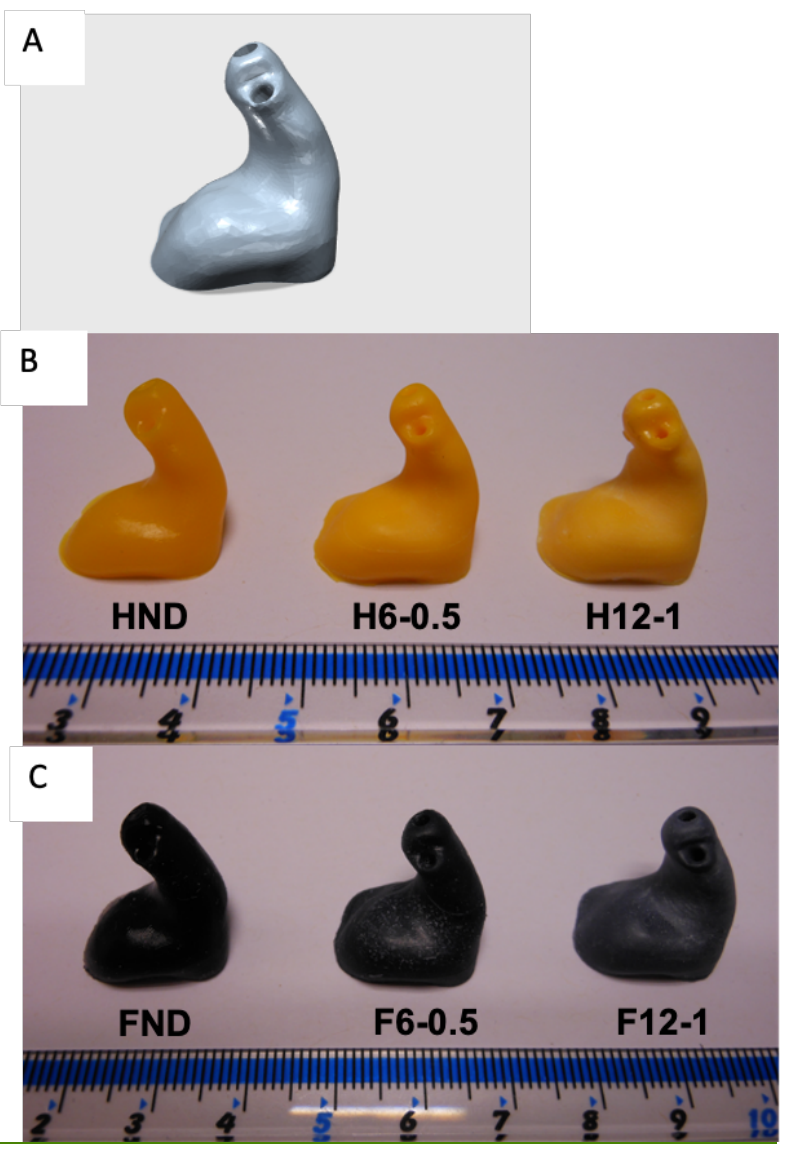

Figure 1. (A) 3D scan model of the hearing aid; DLP 3D printed hearing aids using (B)

ENG hard resin and $(C)$ Flexible resin. In $(B)$ and $(C)$ from left to right, hearing aids fabricated without drug, with ciprofloxacin-fluocinolone acetonide $6 \%-0.5 \%$ and $12 \%$ $1 \%$, respectively. Scale in $\mathrm{cm}$. 

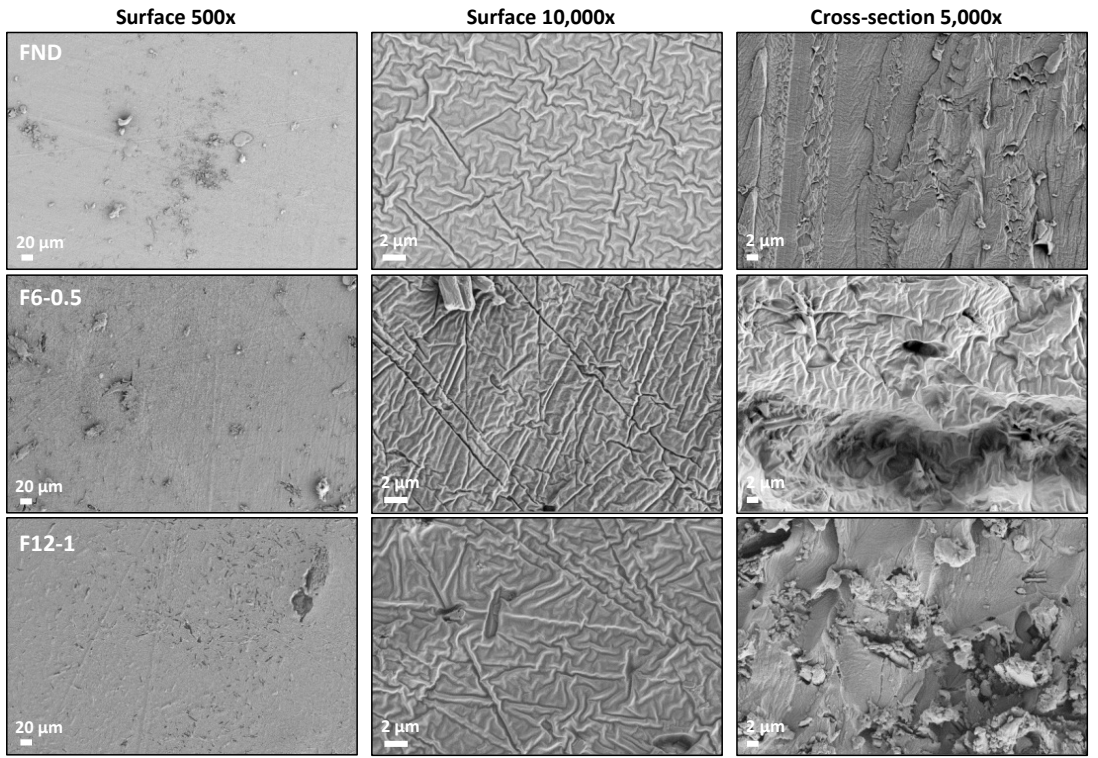

SEM images of the control 3D printed slabs (without drugs) revealed homogeneous matrices lacking pores (Figures 2 and 3). Similar appearance was observed for F6-0.5 and H6-0.5 although with a minor increase in roughness. Conversely, F12-1 and H12-1 showed small particles dispersed inside the slab (cross-section views) and acicular marks on the surface. These empty marks may correspond to drug particles located on the outer surface of the slab that were removed during the washing process with isopropanol.

Figure 2. SEM images of freshly prepared 3D printed slabs FND, F6-0.5 and F12-1. 


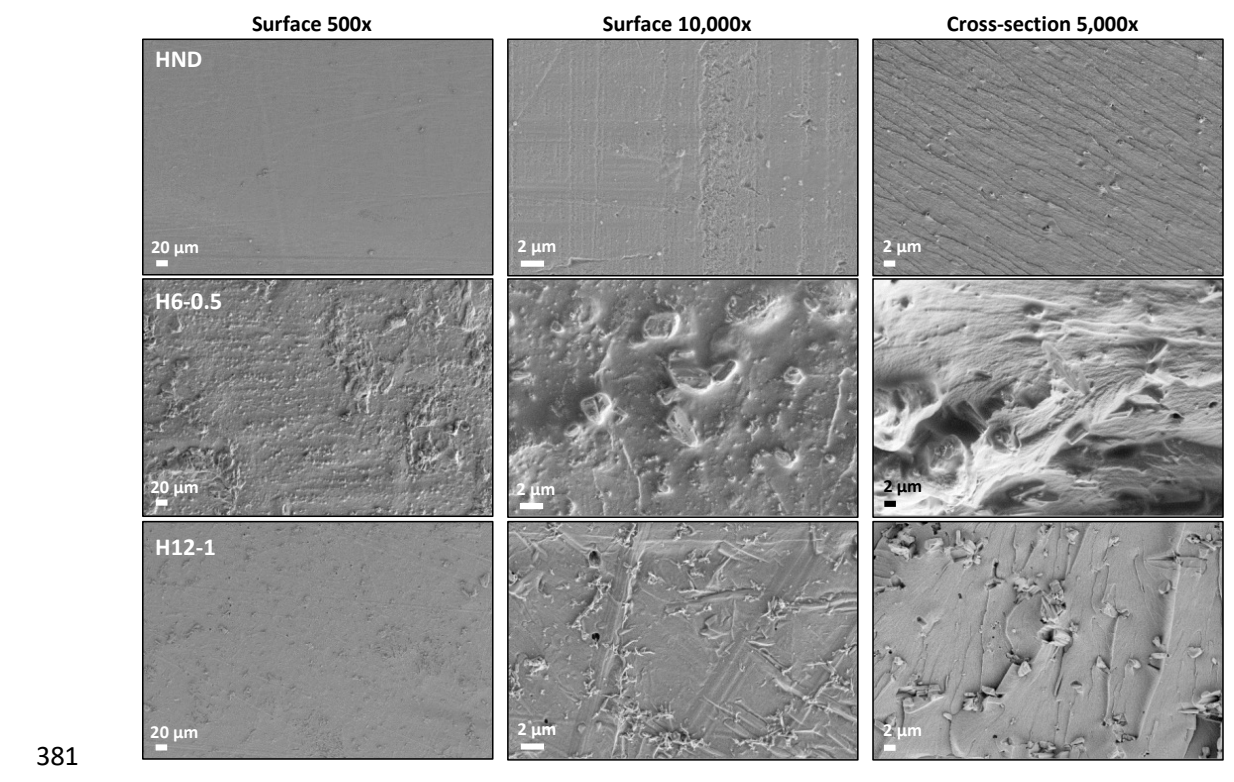

382 Figure 3. SEM images of freshly prepared 3D printed slabs HND, H6-0.5 and H12-1. 
383 To gain an insight into the crystalline structure of the drugs after DLP 3D printing, DSC 384 and XRPD spectra were obtained (Figure 4). FND and HND were amorphous and DSC 385 scans only revealed small decomposition peaks at $177^{\circ} \mathrm{C}$ and $174^{\circ} \mathrm{C}$, respectively. 386 Differently, F12-1 and $\mathrm{H} 12-1$ showed a thermal event at $151^{\circ} \mathrm{C}$, which is the typical dehydration temperature of ciprofloxacin salt (Figure 4A). In good agreement, XRPD spectra of F12-1 and H12-1 also evidenced crystalline peaks of ciprofloxacin at $8.33^{\circ}$, $9.17^{\circ}, 19.44^{\circ}$ and $26.64^{\circ} 2 \theta$ (Figure $4 \mathrm{~B}$ ). Fluocinolone acetonide (less polar and in less amount) seemed to have been dissolved completely in the resins, and no crystalline peaks corresponding to this drug were observed in the slabs. In the case of the polar ciprofloxacin hydrochloride, the resins only allowed the molecular dispersion of a certain proportion of drug (up to ca. $6 \%$ ciprofloxacin in the blend) while the rest of the drug remained as small crystalline particles. Endothermic events typical of ciprofloxacin have also been previously recorded for 3D printed filaments used for FDM for a variety of polymers both when the drug was blended with the components or adsorbed onto preformed filaments $[58,59]$. Reports evidenced that ciprofloxacin is indeed immiscible with the matrix of urinary catheters made of poly(D,L-lactide-co- $\varepsilon$-caprolactone) [60]. 

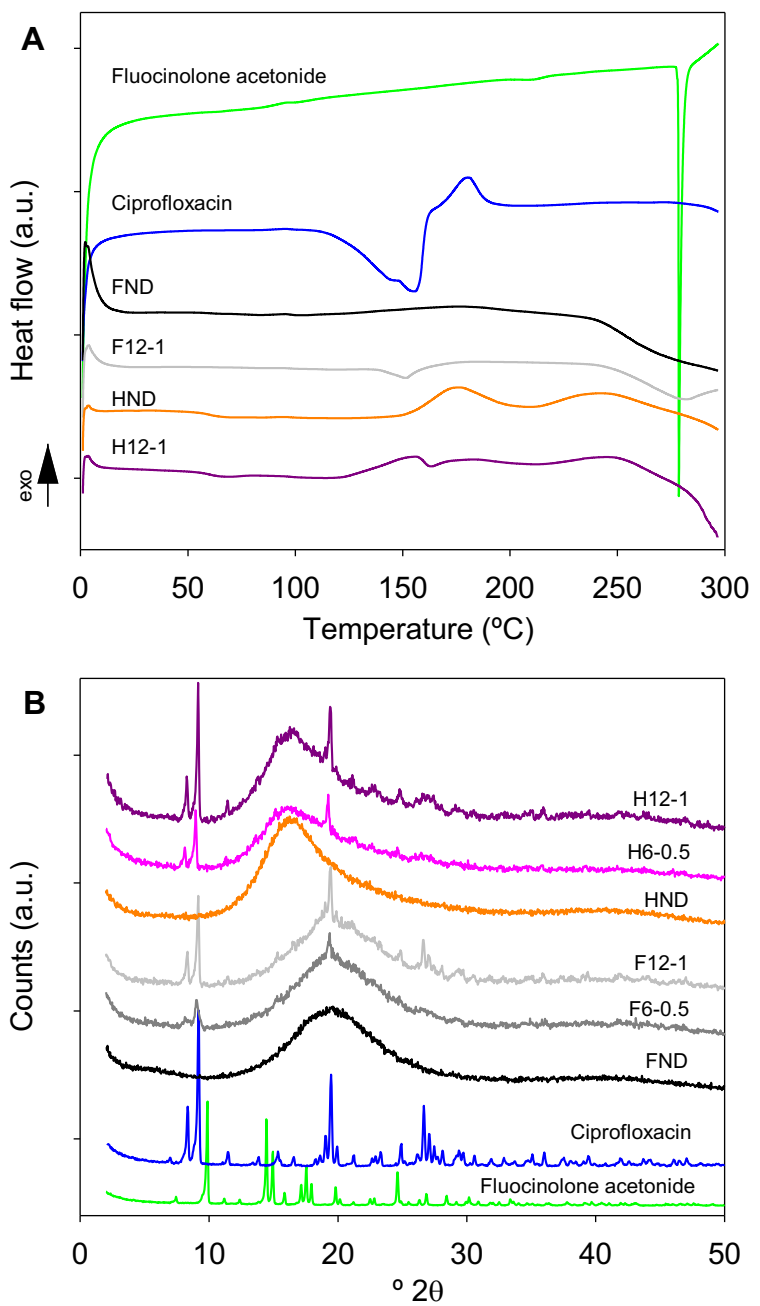

Figure 4. (A) DSC thermograms and (B) XRPD spectra (B) of the drugs, 3D printed slabs FND and HND (without drug) and 3D printed drug-loaded slabs. Codes as in Table 1.

$403 \quad$ X-ray micro-CT imaging was used to three-dimensionally visualise the internal structure 404 of the fabricated hearing aids. As can be seen from Figure 5, the images indicated that 405 hearing aids loaded with drugs had more dense regions (in blue colour) compared with 406 the ones printed without drug. By increasing the drug loading of the devices from $6 \%$ 
ciprofloxacin $-0.5 \%$ fluocinolone acetonide $(\mathrm{w} / \mathrm{w}$ ) to $12 \%-1 \%$, an increased amount of blue colour can be observed. In order to estimate the dimensional accuracy of the 3D printed devices, their dimensions were compared with the dimensions of the 3D model (Table S1, Supplementary Material). The results demonstrated that the measured dimensions were similar to the dimensions in the 3D design except for the slightly higher variability in the $\mathrm{x}$-axis values, indicating the suitable precision and reproducibility of the $3 \mathrm{D}$ printing process. The porosity results showed that all the $3 \mathrm{D}$ printed devices had a total porosity percentage in the range of $0.3 \%-2 \%$, indicating that the devices are not porous.

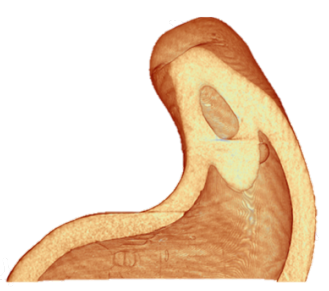

FND

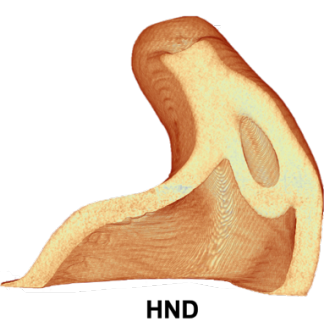

HND

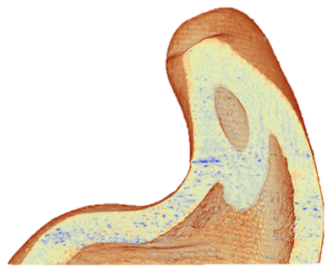

F6-0.5

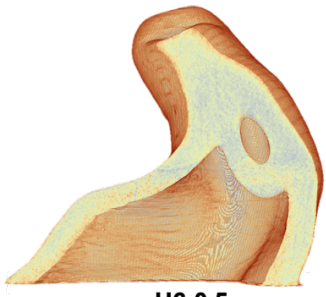

H6-0.5

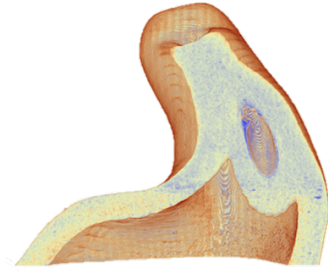

F12-1

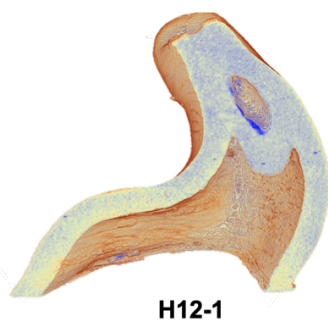

Figure 5. X-ray micro-CT images of the hearing devices. Codes as in Table 1.

\subsection{Mechanical evaluation of the slabs}

Since drug-loaded devices obtained by vat photopolymerization 3D printing have been barely investigated [46], a first step was to elucidate whether the addition of antimicrobial and anti-inflammatory agents at clinically relevant proportions may have an impact on the mechanical properties of the hearing aids. 3D printed FND and HND slabs showed quite different mechanical properties (Figure $\underline{6}$ ). As expected, FND slabs made of Flexible resin were able to deform more than $60 \%$ in length before breaking. The Young's modulus of FND was 2.5 (s.d. 0.2) MPa (Table 2). The addition of ciprofloxacin and fluocinolone acetonide caused a slight increase in the modulus up to 3.7 and $4.1 \mathrm{MPa}$ 
Table 2. Characteristics of the 3D printed slabs. Mean values and, in parenthesis, standard deviations $(n=3)$.

\begin{tabular}{ccc}
\hline Hearing aid code & Young's modulus (MPa) & Hemolysis (\%) \\
\hline FND & $2.5(0.2)$ & $2.3(1.3)$ \\
F6-0.5 & $3.7(0.4)$ & $1.6(0.3)$ \\
F12-1 & $4.1(0.4)$ & $1.9(1.0)$ \\
HND & $152.7(1.8)$ & $1.0(0.3)$ \\
H6-0.5 & $154.0(2.5)$ & $1.7(1.3)$ \\
H12-1 & $155.6(1.9)$ & $1.1(0.9)$ \\
\hline
\end{tabular}

434

On the other hand, HND slabs made of ENG hard resin were more brittle and started to develop cracks at lower strain values. The Young's modulus was remarkably high, 152.7 (s.d. 1.8) MPa. The addition of the drugs made the slabs more brittle, but the Young's modulus remained in the range of 154-156 MPa. Overall, the 3D printed slabs were able to accommodate quite high proportions of drugs while still maintaining their typical mechanical properties.

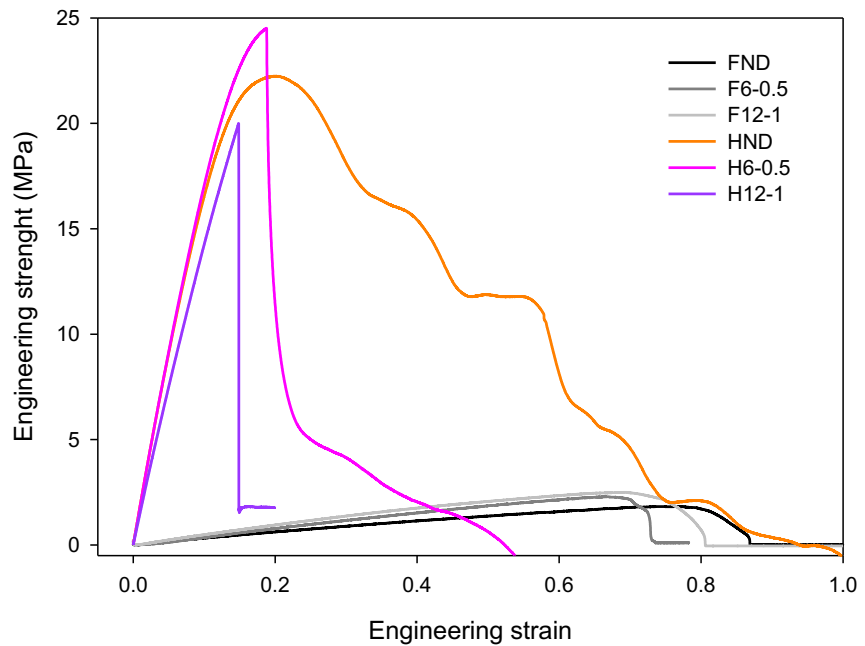


442 Figure $\underline{6}$. Stress-strain curves of the DLP 3D printed slabs as a function of different drug 443 loadings $(n=3)$.

\subsection{Hemolytic activity}

Although hearing aids are not expected to enter into contact with the human blood, ear wounds and infections as well as incorrect handling may expose the device to blood [61]. $3 \mathrm{D}$ printed slabs of each resin with and without drug combinations were evaluated using an agitated blood incubation model [52]. Triton X-100 was used to cause $100 \%$ hemolysis and referred as the positive control. All slabs showed an excellent blood compatibility with hemolysis percentages well below $5 \%$ (Table 2) and no significant differences were found among the formulations (ANOVA, $p=0.5650$ ). This finding also indicated that traces of residual monomers, if any, and the amounts of drugs eluted to the medium do not compromise the safety of the device.

\subsection{Anti-biofilm properties}

All 3D printed slabs showed hydrophilic surface, with water contact angles in the 61-64 range for slabs prepared with the hard resin and in the $63-68^{\circ}$ range for those made with the flexible resin (Figure S3, Supplementary Material). These values were not altered by the incorporation of drugs (ANOVA, $p>0.05$ ).

The anti-biofilm properties of the DLP 3D printed slabs were evaluated against biofilm formation by two bacteria commonly responsible for chronic suppurative otitis media, $P$. aeruginosa and $S$. aureus [62-65]. Drug-loaded and blank devices were tested, and glass coverslips were used as positive controls of bacteria growth. The AAA biofilm growth model was used for testing biofilm formation on different substrate materials with a high reproducibility [53,54]. This setup allowed elucidating whether the drugs (mainly ciprofloxacin) or any other substance is leached from the hearing aids may alter biofilm growth on the surface of the devices. Moreover, bacterial planktonic growth was also monitored in the medium surrounding the $3 \mathrm{D}$ printed slabs in order to assess the degree of leaching of the drugs to the culture medium

In the case of $P$. aeruginosa (Figure $\underline{7}$ ), after 12 hours of incubation, biofilm was successfully developed on the control glass coverslips and also on the blank hearing aids FND and HND (ANOVA, $p=0.3962$ ). Also, no relevant changes in the absorbance of the surrounding growth medium were observed, which means that the planktonic 
475 growth of this Gram-negative bacterium was similar. Therefore, blank hearing aids

476 themselves did not possess biocide surface and did not release bactericide substances

477 to the growth medium. Differently, the drug-loaded devices exhibited an efficient anti478 biofilm activity. The growth on the surface of the devices was completely inhibited in all 479 cases, and the bacteria remaining in the medium was remarkably decreased. The growth 480 of P. aeruginosa in the medium was statistically lower for the drug-loaded hearing aids 481 than for FND, HND and the coverslips (ANOVA, $p<0.0001$ ). No significant differences 482 were observed among F6-0.5, F12-1, H6-0.5 and H12-1, which means that the amount 483 of ciprofloxacin released was much higher than the level required for the antibiofilm 484 effect, reported to be $40 \mu \mathrm{g} / \mathrm{ml}$ for $P$. aeruginosa [66]. 

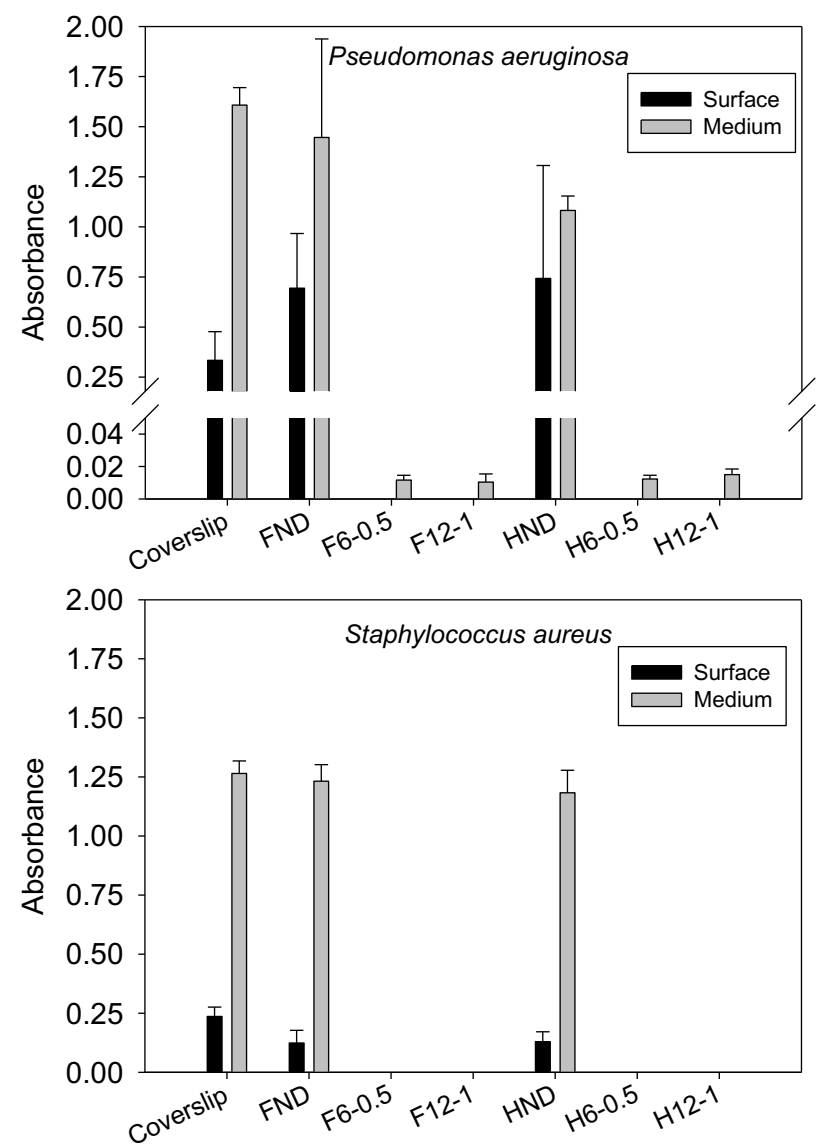

Figure 7 . Growth of $P$. aeruginosa and $S$. aureus on the surface (biofilm) and in the medium surrounding the slabs after 12 and $48 \mathrm{~h}$ incubation, respectively. As controls, glass coverslips were used. Mean values and standard deviations $(n=3)$. The absorbance values were recorded at $570 \mathrm{~nm}$ for the MTT assay (growth on the surface) and at $600 \mathrm{~nm}$ for planktonic bacteria growth in the medium.

In the case of $S$. aureus (Figure 7 ), biofilm formation took more time and therefore it was evaluated after 48 hours of incubation. As observed for $P$. aeruginosa, blank hearing aids performed as the control glass coverslips allowed the development of the Grampositive bacteria biofilm. Drug-loaded devices completely avoided biofilm formation on 
their surfaces and also hindered the bacteria growth in the surrounding medium. The statistical analysis confirmed that the bacteria growth on FND and HND was similar and slightly lower than that recorded for the coverslips (ANOVA, $p=0.0392$ ) while the growth of $S$. aureus in the medium was the same for FND, HND and the coverslips (ANOVA, $\mathrm{p}=0.4514$ ). Minimum bactericidal and biofilm eradication concentrations of ciprofloxacin against methicillin-resistant $S$. aureus have been estimated to be 0.625 and $438 \mu \mathrm{g} / \mathrm{ml}$, respectively [66]. Assuming a weight of $0.15 \mathrm{~g}$ per slab, the amount of ciprofloxacin in the F6-0.5 and H6-0.5 slab would be $9 \mathrm{mg}$. The total volume of culture medium was 4 $\mathrm{ml}$. Therefore, the minimum bactericidal concentration can be reached with the release of only $0.023 \%$ drug content, while the biofilm eradication may require the release of $19.5 \%$ ciprofloxacin content.

The efficacy of ciprofloxacin preventing the biofilm formation of $P$. aeruginosa has been shown before [63-65, 67-69] and was attributed to the inhibition of the Quorum Sensing mechanism [69]. The efficacy against $S$. aureus agreed with previous reports [65, 67] and ciprofloxacin-loaded electrospun nanofibers have demonstrated ability to inhibit the biofilm formation by a methicillin-resistant $S$. aureus [67]. In addition, the fabricated devices showed great stability under the storage condition of room temperature for 3 months without losing the efficacy.

\subsection{Drug release studies}

Since drug-loaded slabs F6-0.5 and H6-0.5 demonstrated to be highly efficient against both Gram-negative and Gram-positive bacteria, only F6-0.5 and H6-0.5 were chosen for a detailed monitoring of the drug release profiles to avoid waste of drug and concerns on toxicity. This information should allow the prediction for how long the hearing aids could continue delivering the drugs, keeping in mind that otitis requires one or two weeks pharmacological treatment [42]. Ciprofloxacin hydrochloride is a hydrophilic antimicrobial agent (solubility $170 \mu \mathrm{g} / \mathrm{mL}$ in PBS; log $\mathrm{P}-0.94$ ) [70], while fluocinolone acetonide is a relatively hydrophobic corticosteroid (solubility $17 \mu \mathrm{g} / \mathrm{mL}$ in PBS; $\log P$ 2.24) [71]. Therefore, the release medium, $50 \%(\mathrm{v} / \mathrm{v})$ ethanol and $50 \%(\mathrm{v} / \mathrm{v})$ water, was chosen to attend a two-fold criterion: i) to generate a less polar environment than water and thus to resemble better the environment in the ear canal (a mixture of sweat and cerumen); and ii) to provide sink conditions for both drugs [72]. Hydroalcoholic medium is commonly used as receptor medium to provide sink conditions in diffusion and release studies of corticosteroid drugs intended for skin application [73]. 
DLP 3D printed drug-loaded discs F6-0.5 and H6-0.5 sustained the releases of both drugs for 20 days and then the releases continued with much slower rates (Figure $\underline{8}$ ). The slowdown of the releases may be attributed to less accessible drug molecules entrapped in the inner regions of the discs. F6- 0.5 provided faster release profiles than H6-0.5. Particularly, the amount of ciprofloxacin released after 23 days from F6-0.5 was 16.5 (s.d. 1.3) mg/g, equivalent to 27.6 (s.d. 2.2) \% loading. In the case of H6-0.5 the cumulative amount of ciprofloxacin released was 1.8 (s.d. 0.2 ) $\mathrm{mg} / \mathrm{g}$, equivalent to 3.0 (s.d. 0.3) \% loading. Similarly, fluocinolone acetonide release occurred faster from F60.5 and reached 4.6 (s.d. 0.1 ) $\mathrm{mg} / \mathrm{g}$ at day 23 , which represent more than $90 \%$ loading (93.1, s.d. 2.3, \%). In the case of $\mathrm{H} 6-0.5$, the amount of fluocinolone acetonide released was 0.52 (s.d. 0.04) mg/g, equivalent to 10.3 (s.d. 0.9) \% loading. Thus, overall, release of fluocinolone acetonide occurred faster and more efficiently than for ciprofloxacin.
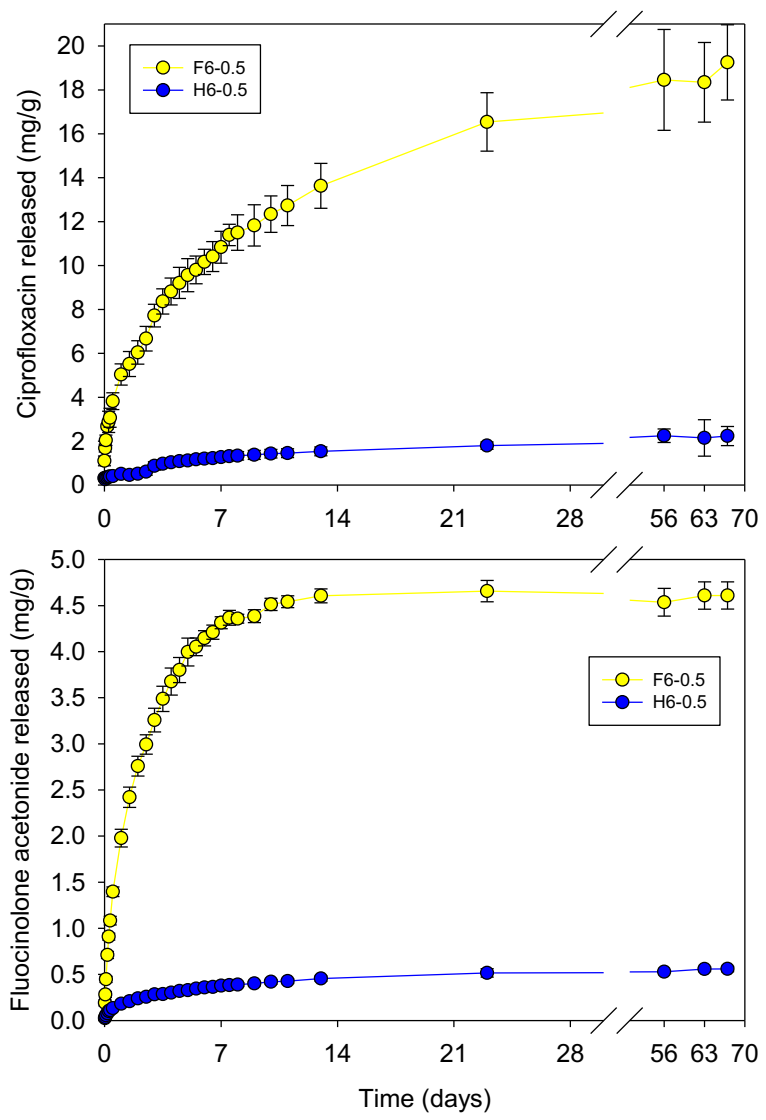
544 Figure $\underline{8}$. Drug release profiles of ciprofloxacin hydrochloride (top) and fluocinolone 545 acetonide (bottom) for 3D printed discs F6-0.5 and H6-0.5.

Release profiles recorded in the first week of release fitted quite well to the square-root kinetics (Table 3, Eq. 3). In the case of ciprofloxacin released from H6-0.5 data recorded for the first two weeks was used for the fitting to obtain reliable values.

Table 3. Results of fitting of Higuchi equation (square root kinetics) to the drug release profiles

\begin{tabular}{ccccc}
\hline \multirow{2}{*}{ Hearing aid code } & \multicolumn{2}{c}{ Ciprofloxacin } & \multicolumn{2}{c}{ Fluocinolone acetonide } \\
& $\mathbf{K}_{\mathbf{H}}\left(\mathbf{m g} \cdot \mathbf{g}^{-1} \cdot \mathbf{h}^{-0.5}\right)$ & $\mathbf{R}^{2}$ & $\mathbf{K}_{\mathbf{H}}\left(\mathbf{m g} \cdot \mathbf{g}^{-1} \cdot \mathbf{h}^{-0.5}\right)$ & $\mathbf{R}^{2}$ \\
\hline F6-0.5 & $0.78($ s.d. 0.06) & $>0.99$ & $0.35($ s.d. 0.01) & $>0.99$ \\
H6-0.5 & 0.083 (s.d. 0.011) & $>0.95$ & 0.028 (s.d. 0.002) & $>0.99$ \\
\hline
\end{tabular}

Drug release rate constants $\left(\mathrm{K}_{\mathrm{H}}\right)$ evidenced that the release of any of the drugs was one order of magnitude faster from the Flexible resins than for the ENG hard resins. Therefore, it is possible to cover a wide range of therapeutic demands by modifying the compositions of the photocurable resin, as observed also for different polymers employed in the fabrication of other 3D printed drug-loaded implants and oral dosage forms [74-76].

After the drug release test, the discs were washed, dried and weighed again. The mean weight loss recorded was $11 \%$ for $\mathrm{F} 6-0.5$ and $1.1 \%$ for $\mathrm{H} 6-0.5$. These values were twofold larger than those predicted from the leakage of the drugs, which suggested that there is dissolution of materials from the slabs after 2 months in the dissolution medium. FND and HND discs stored in the dissolution medium exhibited an appearance like the freshly prepared ones (Figure S2, Supplementary Material). After the dissolution study, the F6-0.5 disc had rougher surface while the H6-0.5 disc showed a variety of pores ranging in size from few nanometers to few microns compared to the freshly prepared ones. This finding suggested that the drug dissolution created channels in the structure that may facilitate partial loss of polymer components. 
Although there are none other 3D printed drug-loaded hearing aids for comparison, the release of more than 7 days for both drugs may satisfy the therapeutic guidelines for the treatment of AOMT using the ciprofloxacin and fluocinolone acetonide combinations [45], which could ensure greater therapeutic compliance and effectiveness compared with ear drops. In addition to the antimicrobial efficacy of ciprofloxacin, the clinical management of otitis has been shown to benefit from the combination with fluocinolone acetonide; the combination being more efficient than ciprofloxacin alone to address the associated edema, otalgia, and otorrhea, and therefore a faster and more complete recovery of the patient [45].

The work highlights the potential of using DLP 3D printing to manufacture drug-loaded hearing aids that required personalization to fit the ear anatomy of the patient. The manufacture approach was easy, less expensive and in just one step. In this scenario, drug could be eluted out from the device via diffusion, providing sustained drug release at local site and hence increasing the residence time. Apart from antibiotics, other agents are possibly to be incorporated for drug delivery to the ear depending on patient's need. Moreover, in order to extend the applicability of our work, cochlear implants may be a potential application to be coupled with DLP 3D printing to the inner ear [77].

\section{Conclusions}

This work demonstrates for first time the possibility of incorporating a combination of ciprofloxacin and fluocinolone acetonide, typically used as drops to address ear infections, into DLP 3D printed hearing aids to prevent or treat biofilm-related infections. Drug-loaded hearing aids were successfully prepared using two types of commercial resins, Flexible and ENG hard with different drug loadings. DLP 3D printed drug-loaded slabs exhibited similar mechanical properties and demonstrated excellent blood compatibility. In vitro drug release studies showed that the devices could be sterilized and were able to provide sustained drug release for more than 7 days for both drugs, satisfying the therapeutic efficacy for the treatment of AOMT. Anti-biofilm properties were evaluated against $P$. aeruginosa and $S$. aureus, showing no bacteria growth on the surface of the devices and decreased growth in the surrounding medium. This proof-ofconcept study shows that vat photopolymerization 3D printing technologies can be a viable technology for the manufacture of drug-loaded medical devices with anti-biofilm properties. The outcome of this work could be translated and extended for the treatment of other diseases with other devices, allowing personalized therapy. 
606 This study was partially funded by MINECO (SAF2017-83118-R), AEI Spain, Xunta de 607 Galicia (ED431C 2016/008, ED431E 2018/08), and FEDER. M. Vivero-Lopez 608 acknowledges Xunta de Galicia (Consellería de Cultura, Educación e Ordenación 609 Universitaria) for a predoctoral research fellowship [ED481A-2019/120]. The authors 610 would like to thank Kudo3D Inc. for providing the free license key to use their Print Job 611 Software. Authors would like to thank the use of RIAIDT-USC analytical facilities.

\section{Data Availability}

614 The raw/processed data required to reproduce these findings are available from the 615 authors upon request. 


\section{References}

[1] A. Goyanes, U. Det-Amornrat, J. Wang, A.W. Basit, S. Gaisford, 3D scanning and 3D printing as innovative technologies for fabricating personalized topical drug delivery systems, J Control Release 234 (2016) 41-48.

[2] S.J. Trenfield, A. Awad, C.M. Madla, G.B. Hatton, J. Firth, A. Goyanes, S. Gaisford, A.W Basit, Shaping the future: recent advances of 3D printing in drug delivery and healthcare, Expert opinion on drug delivery 16(10) (2019) 1081-1094.

[3] J. Banks, Adding value in additive manufacturing: researchers in the United Kingdom and Europe look to 3D printing for customization, IEEE pulse 4(6) (2013) 22-26.

[4] M. Elbadawi, B.M. Castro, F. Gavins, J. Ong, S. Gaisford, G. Perez, A.W. Basit, P. Cabalar, A Goyanes, M3DISEEN: A Novel Machine Learning Approach for Predicting the 3D Printability of Medicines, Int J Pharm (In press) (2020).

[5] M.A. Alhnan, T.C. Okwuosa, M. Sadia, K.W. Wan, W. Ahmed, B. Arafat, Emergence of 3D Printed Dosage Forms: Opportunities and Challenges, Pharm. Res. 33(8) (2016) 1817-1832.

[6] K. Liang, D. Brambilla, J.C. Leroux, Is 3D printing of pharmaceuticals a disruptor or enabler?, Adv Mater 31(5) (2019) 1805680.

[7] C.I. Gioumouxouzis, E. Tzimtzimis, O.L. Katsamenis, A. Dourou, C. Markopoulou, N. Bouropoulos, D. Tzetzis, D.G. Fatouros, Fabrication of an osmotic 3D printed solid dosage form for controlled release of active pharmaceutical ingredients, Eur. J. Pharm. Sci. 143 (2020) 105176.

[8] N. Genina, J.P. Boetker, S. Colombo, N. Harmankaya, J. Rantanen, A. Bohr, Anti-tuberculosis drug combination for controlled oral delivery using 3D printed compartmental dosage forms: From drug product design to in vivo testing, J Control Release 268 (2017) 40-48.

[9] A. Maroni, A. Melocchi, F. Parietti, A. Foppoli, L. Zema, A. Gazzaniga, 3D printed multicompartment capsular devices for two-pulse oral drug delivery, J Control Release 268 (2017) 10-18.

[10] A. Goyanes, C.M. Madla, A. Umerji, G.D. Piñeiro, J.M.G. Montero, M.J.L. Diaz, M.G. Barcia, F. Taherali, P. Sánchez-Pintos, M.-L. Couce, Automated therapy preparation of isoleucine formulations using 3D printing for the treatment of MSUD: First single-centre, prospective, crossover study in patients, Int J Pharm 567 (2019) 118497.

[11] J. Conceição, X. Farto-Vaamonde, A. Goyanes, O. Adeoye, A. Concheiro, H. CabralMarques, J.M.S. Lobo, C. Alvarez-Lorenzo, Hydroxypropyl- $\beta$-cyclodextrin-based fast dissolving carbamazepine printlets prepared by semisolid extrusion 3D printing, Carbohydrate polymers 221 (2019) 55-62.

[12] F. Fina, A. Goyanes, C.M. Madla, A. Awad, S.J. Trenfield, J.M. Kuek, P. Patel, S. Gaisford, A.W. Basit, 3D printing of drug-loaded gyroid lattices using selective laser sintering, Int J Pharm 547(1-2) (2018) 44-52.

[13] A. Awad, F. Fina, S.J. Trenfield, P. Patel, A. Goyanes, S. Gaisford, A.W. Basit, 3D Printed Pellets (Miniprintlets): A Novel, Multi-Drug, Controlled Release Platform Technology, Pharmaceutics 11(4) (2019). 
[14] M. Sadia, B. Arafat, W. Ahmed, R.T. Forbes, M.A. Alhnan, Channelled tablets: An innovative approach to accelerating drug release from 3D printed tablets, J Control Release 269 (2018) 355-363.

[15] A. Isreb, K. Baj, M. Wojsz, M. Isreb, M. Peak, M.A. Alhnan, 3D printed oral theophylline doses with innovative 'radiator-like' design: Impact of polyethylene oxide (PEO) molecular weight, Int. J. Pharm. 564 (2019) 98-105.

[16] A. Awad, A. Yao, S.J. Trenfield, A. Goyanes, S. Gaisford, A.W. Basit, 3D Printed Tablets (Printlets) with Braille and Moon Patterns for Visually Impaired Patients, Pharmaceutics 12(2) (2020) 172.

[17] A. Goyanes, A. Fernández-Ferreiro, A. Majeed, N. Gomez-Lado, A. Awad, A. LuacesRodríguez, S. Gaisford, P. Aguiar, A.W. Basit, PET/CT imaging of 3D printed devices in the gastrointestinal tract of rodents, Int J Pharm 536(1) (2018) 158-164.

[18] S.A. Khaled, J.C. Burley, M.R. Alexander, J. Yang, C.J. Roberts, 3D printing of five-in-one dose combination polypill with defined immediate and sustained release profiles, J Control Release 217 (2015) 308-14.

[19] D.M. Smith, Y. Kapoor, G.R. Klinzing, A.T. Procopio, Pharmaceutical 3D printing: Design and qualification of a single step print and fill capsule, Int J Pharm 544(1) (2018) 21-30.

[20] N. Genina, J. Holländer, H. Jukarainen, E. Mäkilä, J. Salonen, N. Sandler, Ethylene vinyl acetate (EVA) as a new drug carrier for 3D printed medical drug delivery devices, Eur. J. Pharm. Sci. 90 (2016) 53-63.

[21] J. Holländer, N. Genina, H. Jukarainen, M. Khajeheian, A. Rosling, E. Mäkilä, N. Sandler, Three-dimensional printed PCL-based implantable prototypes of medical devices for controlled drug delivery, J. Pharm. Sci. 105(9) (2016) 2665-2676.

[22] J. Fu, X. Yu, Y. Jin, 3D printing of vaginal rings with personalized shapes for controlled release of progesterone, Int J Pharm 539(1-2) (2018) 75-82.

[23] A. Melocchi, N. Inverardi, M. Uboldi, F. Baldi, A. Maroni, S. Pandini, F. Briatico-Vangosa, L. Zema, A. Gazzaniga, Retentive device for intravesical drug delivery based on water-induced shape memory response of poly(vinyl alcohol): design concept and 4D printing feasibility, Int J Pharm 559 (2019) 299-311.

[24] K. Liang, S. Carmone, D. Brambilla, J.-C. Leroux, 3D printing of a wearable personalized oral delivery device: A first-in-human study, Sci Adv 4(5) (2018) eaat2544.

[25] A. Melocchi, M. Uboldi, N. Inverardi, F. Briatico-Vangosa, F. Baldi, S. Pandini, G. Scalet, F. Auricchio, M. Cerea, A. Foppoli, Expandable drug delivery system for gastric retention based on shape memory polymers: Development via 4D printing and extrusion, Int J Pharm 571 (2019) 118700.

[26] X. Farto-Vaamonde, G. Auriemma, R.P. Aquino, A. Concheiro, C. Alvarez-Lorenzo, Postmanufacture loading of filaments and 3D printed PLA scaffolds with prednisolone and dexamethasone for tissue regeneration applications, Eur. J. Pharm. Biopharm. 141 (2019) 100110. 
[27] F.P. Melchels, J. Feijen, D.W. Grijpma, A review on stereolithography and its applications in biomedical engineering, Biomaterials 31(24) (2010) 6121-6130.

[28] X. Xu, A. Awad, P.R. Martinez, S. Gaisford, A. Goyanes, A.W. Basit, Vat photopolymerization 3D printing for advanced drug delivery and medical device applications, J Control Release (Accepted) (2020).

[29] P. Robles-Martinez, X. Xu, S.J. Trenfield, A. Awad, A. Goyanes, R. Telford, A.W. Basit, S. Gaisford, 3D Printing of a Multi-Layered Polypill Containing Six Drugs Using a Novel Stereolithographic Method, Pharmaceutics 11(6) (2019) 274.

[30] X. Xu, P. Robles-Martinez, C.M. Madla, F. Joubert, A. Goyanes, A.W. Basit, S. Gaisford, Stereolithography (SLA) 3D printing of an antihypertensive polyprintlet: Case study of an unexpected photopolymer-drug reaction, Additive Manufacturing 33 (2020) 101071.

[31] C.J. Bloomquist, M.B. Mecham, M.D. Paradzinsky, R. Janusziewicz, S.B. Warner, J.C. Luft, S.J. Mecham, A.Z. Wang, J.M. DeSimone, Controlling release from 3D printed medical devices using CLIP and drug-loaded liquid resins, J Control Release 278 (2018) 9-23.

[32] S.N. Economidou, C.P.P. Pere, A. Reid, M.J. Uddin, J.F. Windmill, D.A. Lamprou, D. Douroumis, 3D printed microneedle patches using stereolithography (SLA) for intradermal insulin delivery, Materials Science and Engineering: C 102 (2019) 743-755.

[33] W. Yao, D. Li, Y. Zhao, Z. Zhan, G. Jin, H. Liang, R. Yang, 3D Printed Multi-Functional Hydrogel Microneedles Based on High-Precision Digital Light Processing, Micromachines 11(1) (2020) 17.

[34] S.H. Lim, J.Y. Ng, L. Kang, Three-dimensional printing of a microneedle array on personalized curved surfaces for dual-pronged treatment of trigger finger, Biofabrication 9(1) (2017) 015010.

[35] Dentca, DENTCA 3D Printed Dentures, 2016. https://www.dentca.com/products/dentca3d. (Accessed 13th April 2020).

[36] EnvisionTEC, The Top Choice for 3D Printed Hearing Aids, Inner-Ear Devices, 2020. https://envisiontec.com/3d-printing-industries/medical/hearing-aid/. (Accessed 21st June 2020).

[37] Materialise, The Hearing-Aid Industry Will Never Be The Same Again, 2020.

https://www.materialise.com/en/cases/phonak-3d-printed-hearing-aid. (Accessed 1st September 2020).

[38] Formlabs, 3D Printing for Audiology, 2020. https://formlabs.com/uk/industries/audiology/. (Accessed 1st September 2020).

[39] Sonova, 3D Printing Technology for Improved Hearing, 2020. https://www.sonova.com/en/story/innovation/3d-printing-technology-improved-hearing. (Accessed 1st September 2020).

[40] WHO, Deafness And Hearing Loss, 2020. https://www.who.int/en/news-room/factsheets/detail/deafness-and-hearing-loss. (Accessed 24th Mar 2020). 
[41] F.T. Orji, E.O. Onyero, C.E. Agbo, The clinical implications of ear canal debris in hearing aid users, Pakistan journal of medical sciences 30(3) (2014) 483.

[42] C.G. Brennan-Jones, K. Head, L.Y. Chong, N. Tu, M.J. Burton, A.G.M. Schilder, M.F. Bhutta, Topical antibiotics for chronic suppurative otitis media, Cochrane Database Syst. Rev. 2018(6) (2018) CD013051.

[43] M.A. Onali, S.B. Bareeqa, S. Zia, S.I. Ahmed, A. Owais, A.N. Ahmad, Efficacy of Empirical Therapy With Combined Ciprofloxacin Versus Topical Drops Alone in Patients With Tubotympanic Chronic Suppurative Otitis Media: A Randomized Double-Blind Controlled Trial, Clinical medicine insights. Ear, nose and throat 11 (2018) 1179550617751907.

[44] Z. Spektor, F. Pumarola, K. Ismail, B. Lanier, I. Hussain, J. Ansley, H.F. Butehorn, K. Esterhuizen, J. Byers, F. Douglis, Efficacy and safety of ciprofloxacin plus fluocinolone in otitis media with tympanostomy tubes in pediatric patients: a randomized clinical trial, JAMA Otolaryngology-Head \& Neck Surgery 143(4) (2017) 341-349.

[45] J. Lorente, F. Sabater, M. Rivas, J. Fuste, J. Risco, M. Gómez, Ciprofloxacin plus fluocinolone acetonide versus ciprofloxacin alone in the treatment of diffuse otitis externa, The Journal of Laryngology \& Otology 128(7) (2014) 591-598.

[46] D.H. Ballard, K. Tappa, C.J. Boyer, U. Jammalamadaka, K. Hemmanur, J.A. Weisman, J.S. Alexander, D.K. Mills, P.K. Woodard, Antibiotics in 3D-printed implants, instruments and materials: benefits, challenges and future directions, Journal of 3D printing in medicine 3(2) (2019) 83-93.

[47] N. Sandler, I. Salmela, A. Fallarero, A. Rosling, M. Khajeheian, R. Kolakovic, N. Genina, J. Nyman, P. Vuorela, Towards fabrication of 3D printed medical devices to prevent biofilm formation, Int J Pharm 459(1-2) (2014) 62-64.

[48] L. Deng, Y. Deng, K. Xie, AgNPs-decorated 3D printed PEEK implant for infection control and bone repair, Colloids and Surfaces B: Biointerfaces 160 (2017) 483-492.

[49] V. Martin, I.A. Ribeiro, M.M. Alves, L. Gonçalves, R.A. Claudio, L. Grenho, M.H. Fernandes, P. Gomes, C.F. Santos, A.F. Bettencourt, Engineering a multifunctional 3D-printed PLAcollagen-minocycline-nanoHydroxyapatite scaffold with combined antimicrobial and osteogenic effects for bone regeneration, Materials Science and Engineering: C 101 (2019) 1526.

[50] M. Kostakioti, M. Hadjifrangiskou, S.J. Hultgren, Bacterial biofilms: development, dispersal, and therapeutic strategies in the dawn of the postantibiotic era, Cold Spring Harbor perspectives in medicine 3(4) (2013) a010306.

[51] J.M. Parrish, M. Soni, R. Mittal, Subversion of host immune responses by otopathogens during otitis media, Journal of Leukocyte Biology 106(4) (2019) 943-956.

[52] M. Weber, H. Steinle, S. Golombek, L. Hann, C. Schlensak, H.P. Wendel, M. Avci-Adali, Blood-contacting biomaterials: in vitro evaluation of the hemocompatibility, Frontiers in bioengineering and biotechnology 6 (2018) 99.

[53] R.A. Exterkate, W. Crielaard, J.M. Ten Cate, Different response to amine fluoride by Streptococcus mutans and polymicrobial biofilms in a novel high-throughput active attachment model, Caries research 44(4) (2010) 372-9. 
[54] C. Mayer, A. Muras, M. Romero, M. López, M. Tomás, A. Otero, Multiple quorum quenching enzymes are active in the nosocomial pathogen Acinetobacter baumannii ATCC17978, Frontiers in cellular and infection microbiology 8 (2018) 310.

[55] N.C. Cady, K.A. McKean, J. Behnke, R. Kubec, A.P. Mosier, S.H. Kasper, D.S. Burz, R.A Musah, Inhibition of biofilm formation, quorum sensing and infection in Pseudomonas aeruginosa by natural products-inspired organosulfur compounds, PLoS One 7(6) (2012) e38492.

[56] T. Nuryastuti, H.C. van der Mei, H.J. Busscher, S. Iravati, A.T. Aman, B.P. Krom, Effect of cinnamon oil on icaA expression and biofilm formation by Staphylococcus epidermidis, Applied and environmental microbiology 75(21) (2009) 6850-6855.

[57] A. Awad, S.J. Trenfield, A. Goyanes, S. Gaisford, A.W. Basit, Reshaping drug development using 3D printing, Drug Discov Today 23(8) (2018) 1547-1555.

[58] N. Qamar, N. Abbas, M. Irfan, A. Hussain, M.S. Arshad, S. Latif, F. Mehmood, M.U. Ghori, Personalized 3D printed ciprofloxacin impregnated meshes for the management of hernia, J. Drug Deliv. Sci. Tec. 53 (2019) 101164.

[59] M. Saviano, R.P. Aquino, P. Del Gaudio, F. Sansone, P. Russo, Poly (vinyl alcohol) 3D printed tablets: the effect of polymer particle size on drug loading and process efficiency, Int J Pharm 561 (2019) 1-8.

[60] J. Fernández, I.A. Ribeiro, V. Martin, O.L. Martija, E. Zuza, A.F. Bettencourt, J.-R. Sarasua, Release mechanisms of urinary tract antibiotics when mixed with bioabsorbable polyesters, Materials Science and Engineering: C 93 (2018) 529-538.

[61] L. Živic, D. Živic, Ear injuries caused by parts of hearing aid, Medicinski Glasnik 8(2) (2011).

[62] A. Bankaitis, What's growing on your patients' hearing aids? A study gives you an idea, The Hearing Journal 55(6) (2002) 48-54.

[63] T. Mansoor, M.A. Musani, G. Khalid, M. Kamal, Pseudomonas aeruginosa in chronic suppurative otitis media: sensitivity spectrum against various antibiotics in Karachi, J Ayub Med Coll Abbottabad 21(2) (2009) 120-3.

[64] K. Ikeda, S. Misawa, T. Kusunoki, Comparative bactericidal activity of four fluoroquinolones against Pseudomonas aeruginosa isolated from chronic suppurative otitis media, BMC Ear, Nose and Throat Disorders 15(1) (2015) 5.

[65] K.M. Nia, G. Sepehri, H. Khatmi, M. Shakibaie, Isolation and antimicrobial susceptibility of bacteria from chronic suppurative otitis media patients in Kerman, Iran, Iranian Red Crescent Medical Journal 13(12) (2011) 891.

[66] M.M. Masadeh, K.H. Alzoubi, W.S. Ahmed, A.S. Magaji, In vitro comparison of antibacterial and antibiofilm activities of selected fluoroquinolones against Pseudomonas aeruginosa and methicillin-resistant Staphylococcus aureus, Pathogens 8(1) (2019) 12.

[67] J.J. Ahire, D.P. Neveling, M. Hattingh, L.M. Dicks, Ciprofloxacin-eluting nanofibers inhibits biofilm formation by Pseudomonas aeruginosa and a methicillin-resistant Staphylococcus aureus, PloS one 10(4) (2015). 
[68] M.A. Aslam, Z. Ahmed, R. Azim, Microbiology and drug sensitivity patterns of chronic suppurative otitis media, Journal of the College of Physicians and Surgeons--Pakistan: JCPSP 14(8) (2004) 459-461.

[69] P. Gupta, S. Chhibber, K. Harjai, Subinhibitory concentration of ciprofloxacin targets quorum sensing system of Pseudomonas aeruginosa causing inhibition of biofilm formation \& reduction of virulence, The Indian journal of medical research 143(5) (2016) 643.

[70] M. Olivera, R. Manzo, H. Junginger, K. Midha, V. Shah, S. Stavchansky, J. Dressman, D. Barends, Biowaiver monographs for immediate release solid oral dosage forms: Ciprofloxacin hydrochloride, J. Pharm. Sci. 100(1) (2011) 22-33.

[71] A. Thakur, R.S. Kadam, U.B. Kompella, Influence of drug solubility and lipophilicity on transscleral retinal delivery of six corticosteroids, Drug Metab. Dispos. 39(5) (2011) 771-781.

[72] R. Sheshala, N.K. Anuar, N.H.A. Samah, T.W. Wong, In vitro drug dissolution/permeation testing of nanocarriers for skin application: a comprehensive review, AAPS PharmSciTech 20(5) (2019) 164.

[73] J.M. Christensen, M.C. Chuong, H. Le, L. Pham, E. Bendas, Hydrocortisone diffusion through synthetic membrane, mouse skin, and Epiderm ${ }^{\mathrm{TM}}$ cultured skin, Archives of drug information 4(1) (2011) 10-21.

[74] W. Kempin, C. Franz, L.-C. Koster, F. Schneider, M. Bogdahn, W. Weitschies, A. Seidlitz, Assessment of different polymers and drug loads for fused deposition modeling of drug loaded implants, Eur. J. Pharm. Biopharm. 115 (2017) 84-93.

[75] P.R. Martinez, A. Goyanes, A.W. Basit, S. Gaisford, Fabrication of drug-loaded hydrogels with stereolithographic 3D printing, Int. J. Pharm. 532(1) (2017) 313-317.

[76] S. Kotta, A. Nair, N. Alsabeelah, 3D printing technology in drug delivery: recent progress and application, Current pharmaceutical design 24(42) (2018) 5039-5048.

[77] S. Plontke, G. Götze, T. Rahne, A. Liebau, Intracochlear drug delivery in combination with cochlear implants, Hno 65(1) (2017) 19-28. 
María Vivero-Lopez ${ }^{1}$, Xiaoyan $\mathrm{Xu}^{2}$, Andrea Muras ${ }^{3}$, Ana Otero ${ }^{3}$, Angel Concheiro ${ }^{1}$,

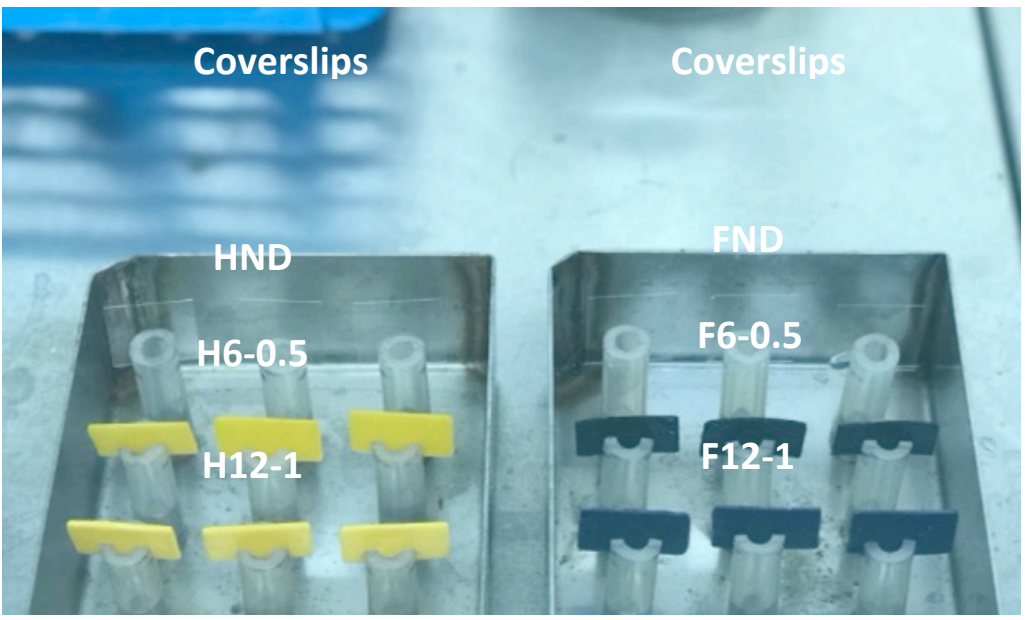

Figure S1. Covers for the AAA-model biofilm cultivation system assembled with the tested materials and coverslips. Covers were placed onto 12-wells cell culture plates

Departamento de Farmacología, Farmacia y Tecnología Farmacéutica, I+D Farma (G 1645), Facultad de Farmacia, and Health Research Institute of Santiago de Compostela (IDIS), Universidade de Santiago de Compostela, 15782 Santiago de Compostela, Spain

${ }^{2}$ Department of Pharmaceutics, UCL School of Pharmacy, University College London, 29-39 Brunswick Square, London, WC1N 1AX, UK

${ }^{3}$ Departamento de Microbiología, Facultad de Biología, Edificio CIBUS, Universidade de Santiago de Compostela, 15782 Santiago de Compostela, Spain

${ }^{4}$ FabRx Ltd., 3 Romney Road, Ashford, Kent TN24 0RW, UK

\section{${ }^{*}$ Corresponding authors}

containing the culture medium inoculated with the biofilm-forming bacteria. 

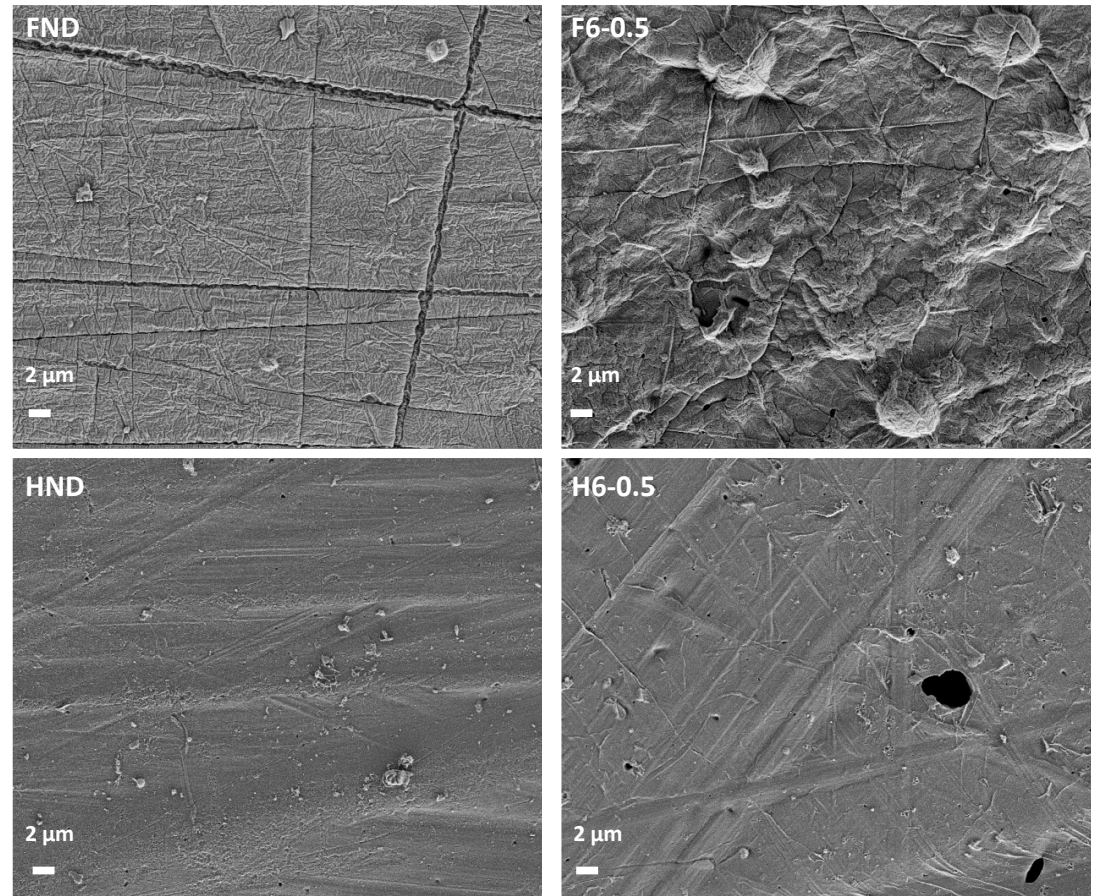

Figure S2. SEM images of 3D printed slabs after being 70 days in the release medium 864 (5000X). Scale bar $2 \mu \mathrm{m}$. 

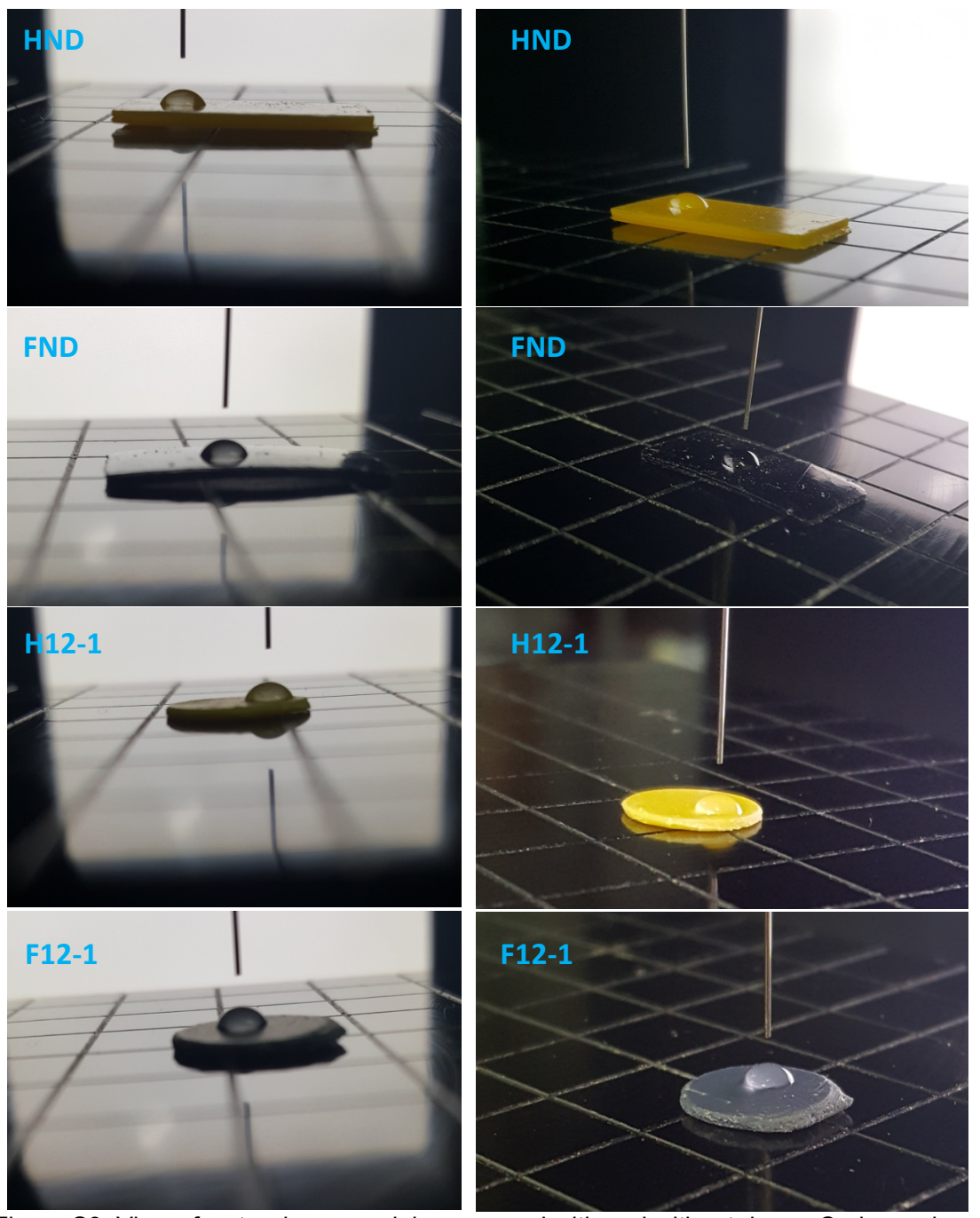
870

Table S1. Dimensions of the 3D model and the 3D printed hearing aids

\begin{tabular}{|c|c|c|c|}
\hline Hearing aid code & $\underline{X(\mathrm{~mm})}$ & $\underline{Y(\mathrm{~mm})}$ & $\underline{Z}(\mathrm{~mm})$ \\
\hline 3D model & 15.82 & 18.66 & 17.68 \\
\hline FND & 17.05 & 18.82 & 17.92 \\
\hline$\underline{F 6-0.5}$ & $\underline{18.70}$ & $\underline{17.83}$ & $\underline{17.59}$ \\
\hline$\underline{F 12-1}$ & $\underline{16.36}$ & $\underline{18.30}$ & $\underline{17.81}$ \\
\hline$\underline{\mathrm{HND}}$ & 16.70 & 18.37 & 16.95 \\
\hline$\underline{\mathrm{H} 6-0.5}$ & $\underline{17.03}$ & $\underline{18.11}$ & $\underline{17.73}$ \\
\hline$\underline{\mathrm{H} 12-1}$ & $\underline{18.08}$ & $\underline{18.18}$ & 17.66 \\
\hline
\end{tabular}

